regulatory proteins through the isolation of Drosophila STATs and by describing their essential role during development. Damell emphasized that this ty pe of experimental approach is crucial to understanding the broader role of this new signaling pathway in gene regulation.

The new trend reflects a more integrated view of how transcription occurs in the context of oher intracellular signaling events. This is party a consequence of recent findings showing that many transcription factors are actually multifunctional complexes that perform a variety of cellular activities. For instance, Danny Reinberg (Robert Wood Johnson Medical School) reponed that mammalian RNA polymerase it consists of a multitude of proteins, including polypetides required for DNA repair (excision and double-strand breaks) and CDK kinases ( 7 and 8 ) required for cellcycle regulation. Rick Young (Whitehead Inst.) described a similar situation for yeast RNA polymerase II, which he referred to as a 'landing dock' for many proteins associated with transcriptional regulation and other cellular activities. Interestingly, among these proteins are members of the Swip/ Snfp complex, which are known to participate in chromosome remodeling via nucleosome disruption. Jim Kadonaga (UC San Diego) described the importance of packaging DNA into chromatin to recreate. in a biochemical reaction, some of the transcriptional phenomena that are observed in vito.

The move towards a more biological view of gene regulation has been facilitated by significant progress in resolving the bior chemical and structural composition of transcription factor complexes. In a very fine session on the structure of DNA-protein complexes, Stephen Burley (Rockefeller Univ.) reported the three dimensional stnicture of a ternary complex containing TBP, TFIIB and DNA as determined by $\mathrm{X}$-ray crystallography, and Mitska Ikura (Ontario Cancer Inst.) showed by NMR spectroscopy that TFIB is a dynamic sucture that has an 'induced fit' upon binding to DNA. But what about the structures of larger multiprotein-DNA complexes? Richard Ebright (Rutgers Univ.) (in collaboration with Reinberg) has been carrying out a systematic analysis of multiprotein - and protein-DNA complexes using biochemical approaches, such as photochemical crosslinking. Ebright described the specific base contacts made by a multi-complex containing TBP, TFIIB, and the three subunits of human TFIIA and DNA. These contacts made by TBP and TFIIB with DNA are remarkably similar to those determined in the X-ray crystallographic structure by Burley, suggesting that this biochemical approach could provide a general strategy to investigate the molecular organization of large multiprotein-DNA complexes.

No transcription meeting would be complete without reports on new factors of biological relevance. Along these lines, Frank Rauscher (Wistar Inst.) reported the isolation and characterization of a new protein that interacts with the KRAB domain, a highly conserved repressor motif that his laboratory has characterized. Rauscher suggested that this co-repressor, which he called 'KRAP' (for KRAB-associated protein), might represent a new class of intermediary molecules linking repressor domains with the transcription initiation complex. Several other co-repressons were described that also interact with certain proteins (or protein domains) to potentiate their repressor actions: Ron Evans (Salk Institute) talked about 'SMRT' (related to $\mathrm{N}-\mathrm{COR}$, described in Jeff Rosenfeld's laboratory), which interacts with steroid hormone receptors; Bob Eisenman (Fred Ilutchinson Cancer Center) describet 'SIN', which interacts with the MAD family (such as MYC and MAX); and Reinberg described a cofactor for DR1 (a general repressor of transcription) that he called DRAP1, which is specifically expressed in post-mitotic cells. As for co-activators, Bob Roeder (Rockefeller Univ.) reported on OCA-B, a cofactor that interacts with OCT1 in certain cell types. Aside from their creative names, these co-factor proteins share many other similar features, including their relatively large size and the presence of multiple functional domains that are presumed to mediate adapter interactions with multiple, different protein factors. Presumably, the next generation of transcriptional regulation will look towards the biological functions of these cofactors. Indeed, even Reinberg, the quintessential biochemist, has begun to examine the physiological consequences of DRAP1 expression in post-mitotic neurons of the cerebral cortex. Alas, the saga continues...

\section{Cory Abate-Shen abate@ocelot.Rutgers.edu \\ Center for Adnanced Biotechnology of Hedicine (CABM). 0́79 Hoes Lane. Piscalatay, NJ 0\$854-5638, USA}

\title{
Cycling in Switzerland
}

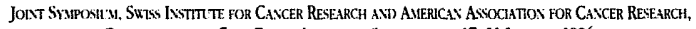

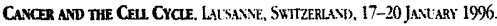

Progresision through the cell cycle is medialed by the sequential activation of a numtwer of cyclins that form active kinase complexes when bound to one of several cyclin-deperdent kinases (CDKs). In G1. these kinases phosphorylate, amongst others, the retinoblastoma protein, which. when phosphorylated, releases the E2F transcription factor, which, in tum. drives cells into $S$ phase. CDKs can be negatively regulated by CKIs (cyclin-dependent kinase inhibitors), which also come in several flavors. Thus, cell-cycle-control proteins either promote division (such as the cyclins, CDKs and E2Fs) or inhibit cell cycling ( $R B$ and $C K I s)$. In this relatively simple view of the cell-cycle work, the former are potential oncogenes, the latter are possible tumor suppressors.

Indeed. David Beach (Cold Spring Hartxor, USA) reported that mice that lack the CKI pl6 develop normally, but are predisposed to a variety of tumors. Primary fibroblasts derived from $\mathrm{pl6}^{--}$mice appear to have an indefinite in vitro lifespan and can be transformed by a Ras oncogene alone (as opposed to wild-type fibroblasts that require a cooperating $M y C$ oncogene).

In contrast, mice that lack the morebroadly acting CKI p27 have a relatively benign phenotype. Jim Roberts (Fred Hutchinson Cancer Center, USA) reported that p27-knockout mice are some $50 \%$ larger than their wild-type littermates, suggesting a quite general growth inhibitory role for $\mathrm{p} 27$. However, benign pituitary tumors developed in p27-deficient animals with almost $100 \%$ penetrance.

Considering the central role of D-type cyclins in cell-cycle progression. mice lacking the cyclin $\mathrm{D} 2$ gene have a surpris- ingly small defect: only the granulosa cells that surround the ovarian follicle fail to proliferate in response to follicle stimulating homone, causing female sterility (Rohert Weinberg. Whitehead Institute, USA).

The most unexpected knockour phenorype was described by Ed Harlow (Massachuserts General Hospital Cancer Center, USA). Mice lacking the E2F1 gene were found to develop tumors of the lungs and reproductive tract between six months and one year of age. In addition, atrophy o! certain tissues was seen, together with a benign lymphoproliferative disease. Thus, even though enforced E2F1 production promotes cell division in vitro, loss of E2FI gene function in vito predisposes to tumorigenesis. This schizcplirenic behavior might be caused by the fact that free E2F1 is a trans-activator, whereas the E2F1-RB 
complex acts as a strong transcriptional repressor. Thus, the loss of E2F1 might lead to the lack of activation of some promoters and derepression of others. The net outcome of E2F1 loss could then differ, depending on the cell type involved. It remains enigmatic why mouse embryonic development can proceed mostly unperturbed in the absence of proteins that appear of critical importance for cell-cycle regulation in vitro.

The first genes that are activated when cells are stimulated with mitogens are named immediate-early genes (examples are $M(y, C, F o s$ and $/ t u)$. The effect of MYC on the cell-cycle machinery was discussed by several speakers. David Beach presented circumstantial evidence that the $C D C 25 \mathrm{~A}$ gene is directly activated by MYC. Robert Eisenman (Fred Hutchinson Cancer Center, USA) described two more MYC target genes, encoding an RNA helicase and a ubiquitin conjugating enzyme with homology to the product of the Drosopbila bendless gene. Martin Eilers (Univ. of Heidelherg, Germany) and Bruno Amati (ISREC, Switzerland) reported that Myc can interfere with the inhibition of cyclin $\mathrm{E}-\mathrm{CDK} 2$ by $\mathrm{p} 27$ through an unidentified mechanism.
Most of the genes that were discussed at this meeting were isolated in the last decade (the retinoblastoma gene will only have its 10th anniversary this year!). I look forward to the cell-cycle meeting of 2006 . I wonder whether anyone will still talk about the genes that caused so much excitement in Lausanne.

\section{René Bernards \\ Bernards@nkinl}

Division of Molecular Carcinogenesis, Netbertands Cancer Institute, Plesmanlaan 121, 1066 CX Amsterdam, The Netberlands.

\title{
International workshop tackles promoter recognition problem
}

\author{
Workshop on Computanonal Analysis of Euknayotic Transcripnonal Regulatoky Elements, DKFZ, HFidflberG, 18-20 JaNuARY 1996.
}

As genome projects evolve, there is a trend towards intense genomic sequencing of poorly characterized regions. This makes the use of computer programs to identify genes and their regulatory regions of increasing importance. In particular, it would be desirable to utilize computational methods not only to identify potential promoter elements, but also to predict the pattem of expression of a gene and, ulitmately, its function. It is with these goals in mind that a group of computer scientists, molecular and structural biologists met at the German Cancer Research Centre (DKFZ) in Heidelberg. for this intemational workshop.

In their opening emarks, the workshop organizers Philipf Bucher (Univ. de Lausanne, Switzerland) and James Fickett (Los Alamos, USA) discussed the communication problems that exist between computational and experimental biologists. Biologists are still using outdated methods, such as searching for consensus sequences, and some of the best computational techniques have limited availability to the bench scientist. By bringing together specialists from different fields, the meeting sought to promote further research and to improve the transfer of techniques from the realm: of theory to that of practical application.

From a personal viewpoint, the meeting provided some stimulating alternative ways to look at DNA sequence. There is a tendency among computational biologists to view DNA as a one-dimensional string of characters. We need to be occasionally reminded that DNA is a molecule, with oher properties besides the sequence of hases. Gert Vriend (EMBL, Heidelberg) provided illustrations of the three-dimensional interaction between protein and DNA, including several beautiful slides showing the bending of DNA by TATAbinding protein and the structure of the Escherichia coli DNA polymerase (Pol) III.

Several presentations centred around the role of chromatin structure in transcription regulation. The location oi nucleosomes might play a large part in determining the accessibility of DNA segments to transcriptional binding factors. Nicolas Mermod (Univ. de Liusanne, Switzerland) provided evidence that transcripticnal binding factors might be directly interacting with histone $\mathrm{H}_{3}$. Unfortunately, there are no current reliable computational means with which to predict nucleosome location.

DNA within living organisms exists in a superhelical conformation, and another factor determining the sites at which tran. scription factors interact is the degree to which the DNA is unwound or destabilized within a promucer region. Craig Benham (Mount Sinai School of Medicine, New York, USA) showed how the global properties of a sequence, operating within a large domain of sequence rather than a small segment, can determine those areas of DNA that destabilize. Artemis Hatzigeorgiou (DKFZ, Heidelberg. Germany), a co-organizer of the meeting. presented work on using neural networks to identify Pol II promoter sequences, and others presented fresh statistical methods and the potential application of 'fuzzy logic' to the identification of promoters.

In the plenary session, researchers generally agreed that the common practice of identifying transcription-factor-binding sites by individual consensus sequence or even with matrix methods is inadequate. For algorithms to he accurate, they must incorporate information regarding the context in which a putative binding site exists, including its location with respect to other potential sites. Global and local properties of the sequence could both be important, as well as the three-dimensional structural features of the molecule and nucleosome location.

Biologists seeking the best currently available techniques should look at two locations on the internet. Dan Prestridge (Univ. of Minnesota, USA) has UNIX and DOS versions of his PROMOTER SCAN program (available at ftp://biosci.umn. edu/pub/proscan/). Thomas Wemer and his colleagues (AG GIODV, GSF, Munich, Germany) provide three programs MatInspector, Consinspector and GenomeInspector (at the web site http://www.gsf. de/biodv/).

Gordon Hutchinson

hutchonetshop.bc.ca

Deparment of Medical Genetics, University of British Columbia, Canada.

\section{Letters to the Editor}

We welcome letters on any topic of interest to geneticists and developmental biologists. Write to:

Dr Mark Patterson, Trends in Genetics,

Elsevier Trends Journals, 68 Hills Road,Cambridge, UK CB2 1LA. 